\title{
Photoacoustic tomography of water in biological tissue
}

\section{Zhun Xu, Changhui Li, Lihong V. Wang}

Zhun $\mathrm{Xu}$, Changhui $\mathrm{Li}$, Lihong V. Wang, "Photoacoustic tomography of water in biological tissue," Proc. SPIE 7899, Photons Plus Ultrasound: Imaging and Sensing 2011, 789912 (17 February 2011); doi: 10.1117/12.873415

SPIE. Event: SPIE BiOS, 2011, San Francisco, California, United States 


\title{
Photoacoustic tomography of water in biological tissue
}

\author{
Zhun Xu, Changhui Li, and Lihong V. Wang* \\ Optical Imaging Laboratory, Dept. of Biomedical Engineering, Washington University in St. Louis, \\ 1 Brookings Dr., St. Louis, MO, USA 63130 \\ *Corresponding author. Email: $\underline{\text { lhwang@biomed.wustl.edu }}$
}

\begin{abstract}
As an emerging imaging technique that combines high optical contrast and ultrasonic detection, photoacoustic tomography (PAT) has been widely used to image optically absorptive objects in both human and animal tissues. PAT overcomes the depth limitation of other high-resolution optical imaging methods, and it is also free from speckle artifacts. To our knowledge, water has never been imaged by PAT in biological tissue. Here, for the first time, we experimentally imaged water in both tissue phantoms and biological tissues using a near infrared (NIR) light source. The differences among photoacoustic images of water with different concentrations indicate that laser-based PAT can usefully detect and image water content in tissue.
\end{abstract}

Keywords: Photoacoustic tomography, water, absorption spectroscopy, blood oxygen saturation

\section{INTRODUCTION}

Water is a very important part in our body. The concentration of water in tissue may reflect both physiological and pathological properties. Some techniques have been used to detect and measure water content in tissue, such as Magnetic Resonance Imaging (MRI) ${ }^{1}$, diffusion-weight MRI (DW-MRI) ${ }^{2}$, and diffuse optical methods. Diffuse optical spectroscopy (DOS) is also selected to measure the spectral changes due to bound water. Although DOS is accurate and sensitive to water state, its spatial location is poor ${ }^{3}$.

Photoacoustic (PA) tomography (PAT) has demonstrated its ability to monitor biological functions. Since optical scattering in biological tissues dominates compared with ultrasound scattering, PAT is capable of providing high resolution imaging at depths beyond the optical transport mean free path ${ }^{4}$. Moreover, PA benefits from a high sensitivity to optical absorption. PA has been applied to image various tissues ${ }^{5}$, and image contrast has been provided by many endogenous and exogenous optical absorbers, such as hemoglobin, melanoma, and various natural and artificial contrast agents. However, to our knowledge, water has never been used as a contrast in PA. Here we will show some initial results of water detection and imaging in phantom, tissue, and in vivo experiment, exploring the potential of laser-based PA application to water. Since there is a local peak at $975 \mathrm{~nm}$ of water absorption spectrum 6 , all of our experiments were done around that wavelength.

\section{METHODS}

\subsection{Experimental setup}

Fig. 1 shows our experimental setup for the water spectral measurement and water content imaging. In Fig.1 (a), the light comes out from the OPO tunable laser. After transmitting through the prism and the ground glass, it finally illuminates on the object (here the object is the water-ethanol mixture as shown in section 2.2). The transducer was put beneath the object to receive the PA signal. The signal was amplified and then delivered to the computer. Fig. 1 (b) shows the experimental setup for the photoacoustic tomography. Here we used our latest real-time imaging system ${ }^{7}$. The ring array with 512 elements was inserted in the system in order to speed up the image acquirement.

Photons Plus Ultrasound: Imaging and Sensing 2011, edited by Alexander A. Oraevsky, Lihong V. Wang, Proc. of SPIE Vol. 7899, 789912 · (C) 2011 SPIE · CCC code: 1605-7422/11/\$18 · doi: 10.1117/12.873415 


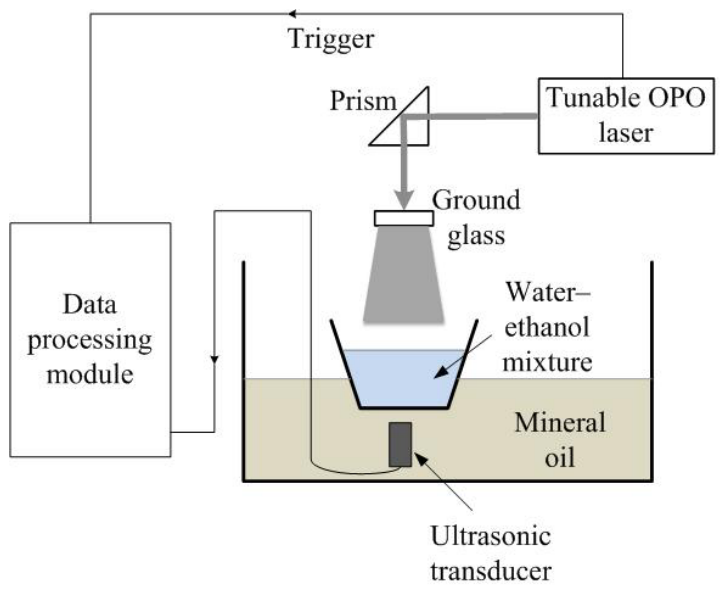

(a)

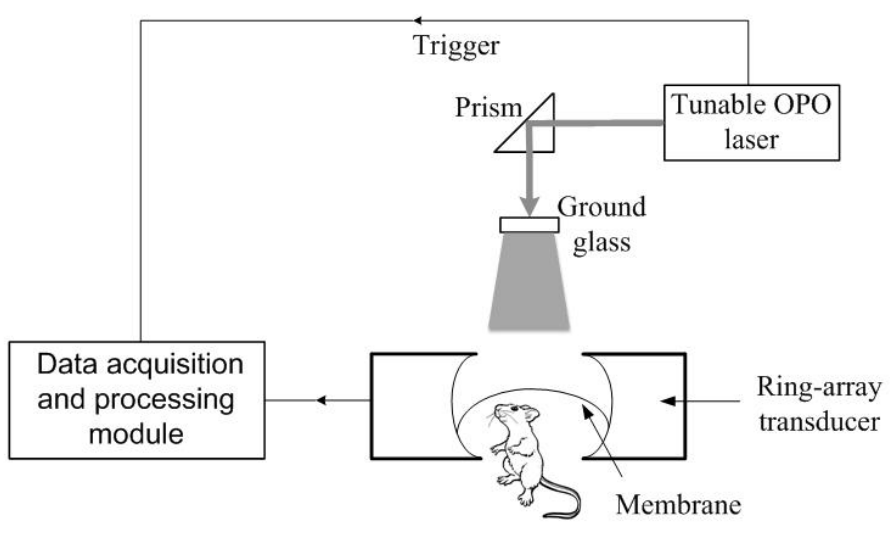

(b)

Figure 1. Schematic of the experimental setups. (a) The schematic of the experimental system for spectral measurement of water-ethanol mixture. (b) The real time ring-array based photoacoustic tomography system for the water imaging experiment.

\subsection{Spectral measurement of water-ethanol mixture}

The generation and propagation of a photoacoustic pressure wave in an acoustically homogenous and non-viscid infinite medium can be described as ${ }^{8,9}$ :

$$
\left(\nabla^{2}-\frac{1}{v_{s}^{2}} \frac{\partial^{2}}{\partial t^{2}}\right) p(\mathbf{r}, t)=-\frac{\beta}{C_{p}} \frac{\partial}{\partial t} H(\mathbf{r}, t)
$$

where $p(\mathbf{r}, t)$ denotes the acoustic pressure at position $\mathbf{r}$ and time $t, v_{s}$ is the speed of sound in the medium, $\beta$ denotes the thermal coefficient of volume expansion, $C_{p}$ denotes the isobaric specific heat capacity, and $H(\mathbf{r}, t)$ is the heating function defined as the thermal energy converted at $\mathbf{r}$ and $t$ per unit volume and time. For optical absorption, the heating function generally equals $\eta_{t h} \mu_{a} \Phi(\mathbf{r}, t)$, where $\eta_{t h}$ is the percentage of energy that is converted into heat that is approximated to one in many cases, $\mu_{a}$ is the optical absorption coefficient, and $\Phi$ is the optical fluence rate.

In general, the initial pressure rise $p_{0}$ at $\mathbf{r}$ immediately after illumination by a short laser pulse is given ${ }^{10}$ :

$$
p_{0}(\mathbf{r})=\Gamma(\mathbf{r}) \eta_{t h} \mu_{a}(\mathbf{r}) F(\mathbf{r}) \text {, }
$$

where $\Gamma=\frac{\beta v_{s}^{2}}{C_{p}}$ is defined as the Grueneisen coefficient (dimensionless), and $F$ is the optical fluence. The initial pressure $p_{0}(\mathbf{r})$, serving as the source of the propagating PA pressure wave, depends on not only the illumination fluence and absorption coefficient, but also on the Grueneisen coefficient. In addition, the absorption coefficient is generally spectrally dependent. After calculating the Grueneisen coefficient based on the corresponding parameters from literatures ${ }^{14}$, we could estimate the optical absorption coefficient of each mixture. The deionized water and waterethanol mixture with $40 \%$ water concentration were used as the samples.

\subsection{Imaging water content in phantoms and tissue}

Two samples were imaged using this PAT system. For sample 1, 3 gel cylinders made of the agar-water mixture $(1 \%, 2 \%$ and $4 \%$ agar respectively) was used. For sample 2 , a piece of porcine fat was used. A hole was drilled in the fat and filled with $2 \%$ agar gel ${ }^{11}$. 


\subsection{In-vivo imaging of cold-induced cerebral edema}

We also tried the in vivo image of water accumulation in the mouse brain, that is, the cerebral edema. We did this experiment with our real-time ring array-based photoacoustic tomography system. The ring array transducer has 512 elements in order to speed up the image acquirement. Each element has the central frequency of $5 \mathrm{MHz}$. the liquid nitrogen was put in to a metal tube, and the tube was put on the mouse head for $\sim 30$ s. Thus the cold injury-induced cerebral edema was generated ${ }^{12}$. Then we used the PAT to monitor the changes in the mouse brain. During the experiment, both the scalp and the skull were kept intact.

\section{RESULTS AND DISCUSSION}

\subsection{Measurement of the absorption spectra of water}

Fig. 2 shows the spectra measurement with PA. Here we used spectrophotometric measurement as the golden standard. The relative absorption spectra were computed with Eq. (2) and then converted to absolute values (solid lines in Fig. 2) by calibrating with the absorption coefficient of deionized water at $975 \mathrm{~nm}$ measured by a spectrophotometer (Cary 5E, Varian, Walnut Greek, CA). The comparison shows good accordance.

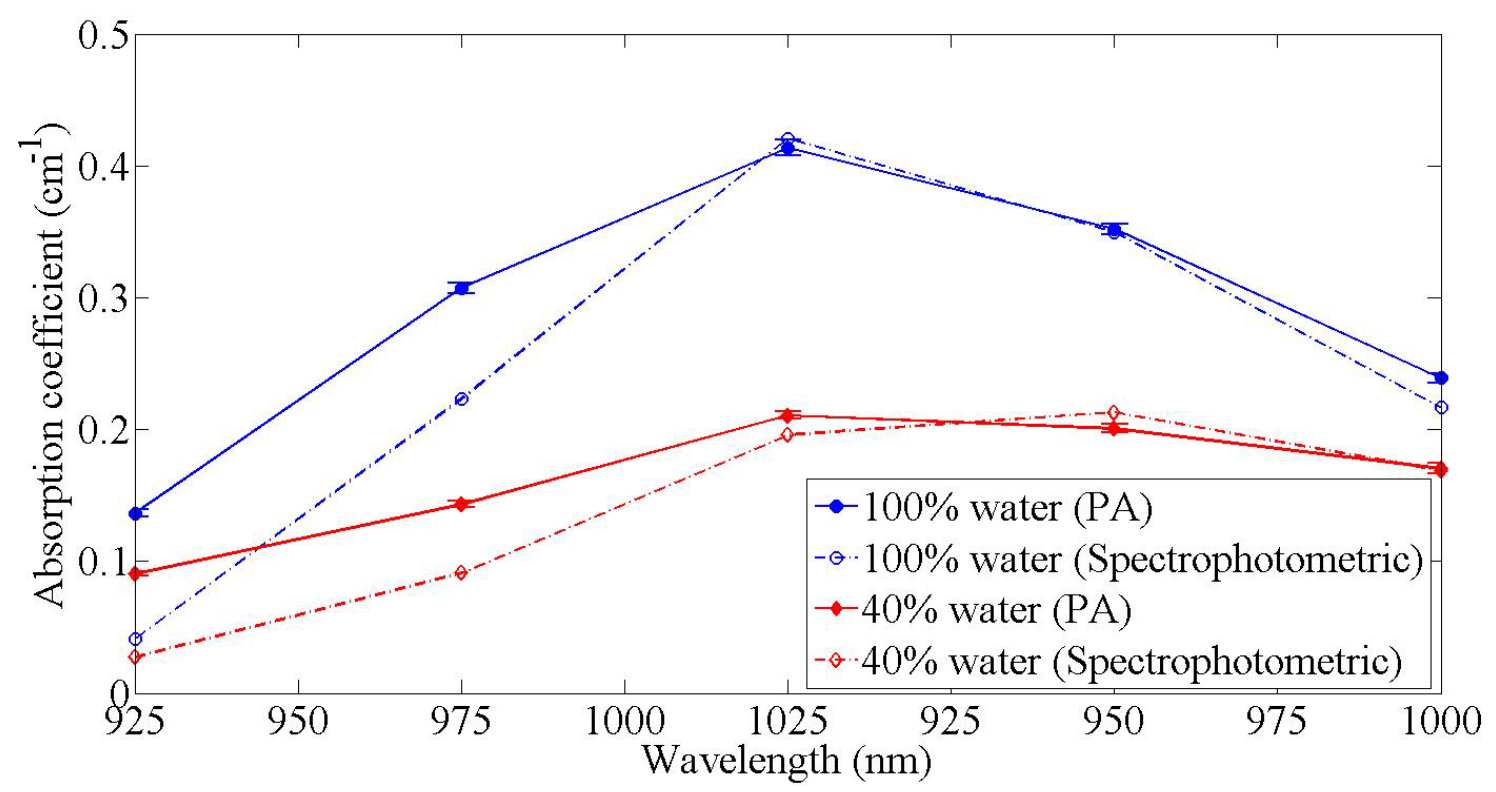

Figure 2. Optical absorption coefficients from photoacoustic (5 means. For each mean, 100 PA signals were used.) and spectrophotometric measurements.

\subsection{Water content imaging in both phantom and tissue experiments}

Fig. 3 shows the result of water content imaging in phantom and tissue experiment. The negative value is partially due to the limited bandwidth of the ultrasonic detection system. From the result we could see that there is a very large contrast between water and fat. Also, we could differentiate the water content with different concentrations in the mixture. 

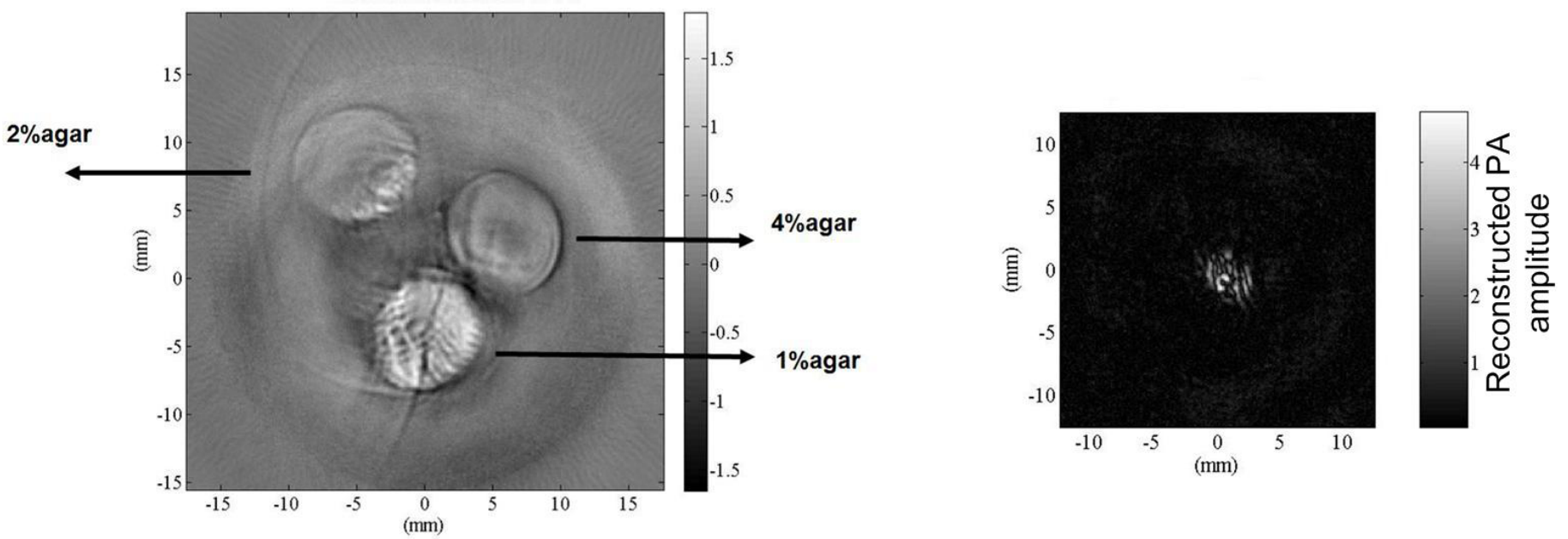

Figure 3. PA image in the phantom and tissue experiment. Left: $1 \%, 2 \%$ and $4 \%$ agar-water mixture in the mineral oil; right: $4 \%$ agar-water mixture was put in the pork fat tissue.

\subsection{In vivo experiment on mouse cerebral edema imaging with PAT}

Fig. 4 shows the result of the brain signal. The images of upper row were obtained at $610 \mathrm{~nm}$, at which wavelength the hemoglobin is the main contrast, so the images mainly reflected the changes of the blood vessel signals. The images of lower row were acquired at $975 \mathrm{~nm}$, at which wavelength we could observed the changes of water signals in the brain. From the figure we could see that there was a decrease of blood flow ${ }^{13}$ but an increase of water accumulation within the first $24 \mathrm{hrs}$ after the cold injury. However, at $36 \mathrm{hrs}$ the mouse began to recover, so the signal, especially the signal of blood vessel came back compared with the original one.

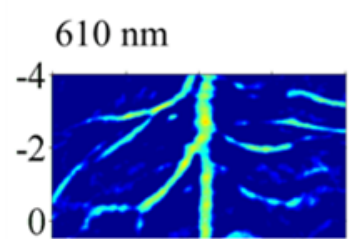

$975 \mathrm{~nm}$

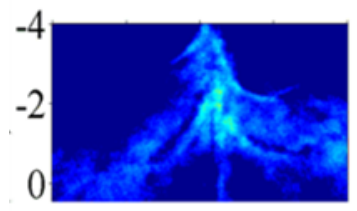

0 hour

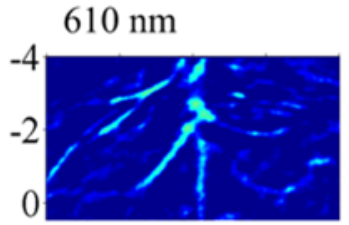

$975 \mathrm{~nm}$

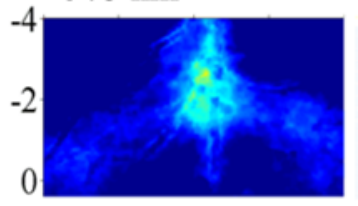

12 hours

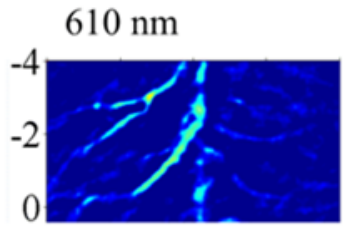

$975 \mathrm{~nm}$

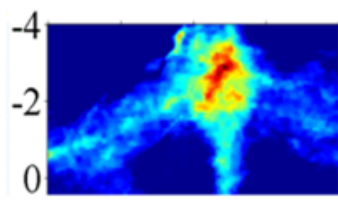

24 hours

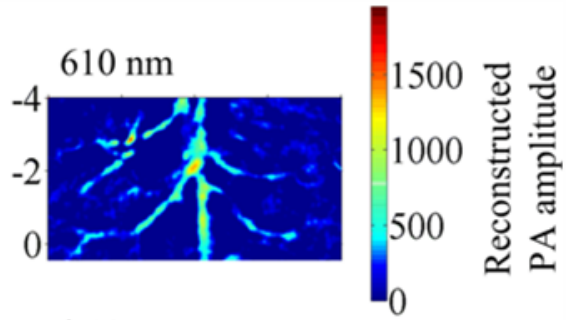

$975 \mathrm{~nm}$

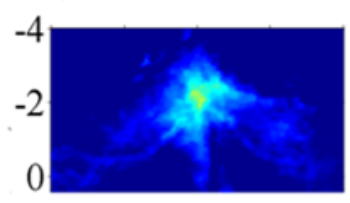

300

200

100

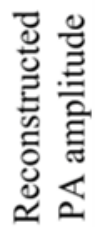

Figure 4. Brain images after cold injury. The images of upper row were acquired at $610 \mathrm{~nm}$, and the ones of lower row were obtained at $975 \mathrm{~nm}$. Maximum water accumulation was observed 24 hours after the cold injury. 


\section{CONCLUSIONS}

To our knowledge, we first detected and imaged the water content in biological tissues ${ }^{14}$. Compared to the water imaging with MRI, PA has the potential to provide higher resolution, and it is faster and less expensive. In the future, we'd explore to image the tissue with different water concentration, especially image the water content in the tissue with low water concentration, such as one of our experiments on water in the fat. Besides, based on the literature ${ }^{3}$, there is a spectral shift between bound water and free water, which could also be implemented in our water imaging. Furthermore, with the results shown above, we have the potential to accurately monitor various physiological parameters, including oxygen saturation, hemoglobin concentration and water concentration. We could differentiate all these parameters by applying multi-wavelengths.

\section{ACKNOWLEDGEMENTS}

This work was sponsored in part by National Institutes of Health grants R01 EB000712, EB000712A2S1, R01 EB00071207S2, R01 EB008085, R01 CA113453901, U54 CA136398, and 5P60 DK02057933. L.W. has a financial interest in Microphotoacoustics, Inc. and Endra, Inc., which, however, did not support this work.

\section{REFERENCES}

[1] D. J. Brooks, P. Luthert, D. Gadian and C. D. Marsden, "Does Signal-Attenuation on High-Field T2-Weighted MRI of the Brain Reflect Regional Cerebral Iron Deposition - Observations on the Relationship between Regional Cerebral Water Proton T2 Values and Iron Levels," Journal of Neurology, Neurosurgery and Psychiatry 52(1), 108-111 (1989)

[2] P. J. Basser, J. Mattiello and D. Lebihan, "Mr Diffusion Tensor Spectroscopy and Imaging," Biophysical Journal 66(1), 259-267 (1994)

[3] S. H. Chung, A. E. Cerussi, C. Klifa, H. M. Baek, O. Birgul, G. Gulsen, S. I. Merritt, D. Hsiang and B. J. Tromberg, "In vivo water state measurements in breast cancer using broadband diffuse optical spectroscopy," Physics in Medicine and Biology 53(23), 6713-6727 (2008)

[4] C. H. Li and L. H. V. Wang, "Photoacoustic tomography and sensing in biomedicine," Physics in Medicine and Biology 54(19), R59-R97 (2009)

[5] L. H. V. Wang, "Prospects of photoacoustic tomography," Medical Physics 35 (12), 5758-5767 (2008)

[6] G. M. Hale, M. R. Querry, "Optical constants of water in the $200 \mathrm{~nm}$ to $200 \mu \mathrm{m}$ wavelength region," Appl. Opt.,12, 555-563 (1973)

[7] C. H. Li, A. Aguirre, J. Gamelin, A. Maurudis, Q. Zhu and L. V. Wang, "Real-time photoacoustic tomography of cortical hemodynamics in small animals," Journal of Biomedical Optics 15(1), - (2010)

[8] P. M. Morse and K. U. Ingard, Theoretical acoustics, McGraw-Hill, New York, (1968)

[9] G. J. Diebold, T. Sun and M. I. Khan, "Photoacoustic Monopole Radiation in 1-Dimension, 2-Dimension, and 3Dimension," Physical Review Letters 67(24), 3384-3387 (1991)

[10] L. V. Wang, "Tutorial on photoacoustic microscopy and computed tomography," Ieee Journal of Selected Topics in Quantum Electronics 14(1), 171-179 (2008)

[11]C. Kim, A. Garcia-Uribe, S.-R. Kothapalli and L. V. Wang, "Optical phantoms for ultrasound-modulated optical tomography," in Design and Performance Validation of Phantoms Used in Conjunction with Optical Measurements of Tissue, pp. 68700M-68708, SPIE, San Jose, CA, USA (2008)

[12] T. D. Oury, C. A. Piantadosi and J. D. Crapo, "Cold-Induced Brain Edema in Mice - Involvement of ExtracellularSuperoxide Dismutase and Nitric-Oxide," Journal of Biological Chemistry 268(21), 15394-15398 (1993)

[13]H. J. Frei, Wallenfa.T, W. Poll, H. J. Reulen, R. Schubert and M. Brock, "Regional Cerebral Blood-Flow and Regional Metabolism in Cold Induced Edema," Acta Neurochirurgica 29(1-2), 15-28 (1973)

[14]Z. Xu, C. H. Li, and L. V. Wang, "Photoacoustic tomography of water in phantoms and tissue," Journal of Biomedical Optics 15 (3), 036019 (2010) 